\title{
Residual Cancer Burden Class 0
}

National Cancer Institute

\section{Source}

National Cancer Institute. Residual Cancer Burden Class O. NCI Thesaurus. Code C160725.

No traces of residual disease; lowest chance of disease recurrence. 